\title{
Sublingualtherapie mit Allergoiden als neue Option
}

in bedeutender Fortschritt in der nach Ansicht von Prof. Dr. Giovanni Passalacqua aus Genua, Italien, mit der Entwicklung carbamylierter monoider Allergenextrakte, sogenannter monomerer Allergoide oder Monoide. Diese sind unter dem Produktnamen Lais ${ }^{\circledR}$ zur sublingualen Therapie in Tabletten- und Tropfenform oder zur subkutanen Therapie auch als Injektionssuspension in Deutschland erhältlich.

Bei der Herstellung der Monoide wird eine Carbamylierung der LysinGruppen durchgeführt. Die so entstandenen Moleküle weisen eine verringerte IgE-Bindungskapazität bei erhaltener Immunogenität auf und zeichnen sich im Vergleich zu anderen Allergoiden nicht zuletzt durch eine geringere Größe und eine hohe enzymatische Resistenz aus. Dies ermöglicht eine einfachere Absorption durch die orale Mukosa und erhöht die Stabilität gegenüber den Speichelenzymen - zwei Gründe, die einen Einsatz der Monoide vor allem zur sublingualen Therapie nahelegen.

Dr. Peter Eberle, pädiatrischer Allergologe und Pneumologe aus Kassel, fasste die Studiendaten zum Einsatz von Lais ${ }^{\circledR}$ in der Pädiatrie zusammen. Er berichtete, dass das Monoid in Studien bereits an mehr als 500 Kindern geprüft worden ist. Zwei wichtige Untersuchungen stellte er im Detail vor: Ein Team um Prof. Dr. Carlo Caffarelli behandelte 48 Kinder (Durchschnittsalter acht Jahre) mit allergischer Rhinokonjunktivitis und Asthma aufgrund einer Sensibilisierung gegen Gräserpollen präsaisonal entweder mit einer sublingualen Im-

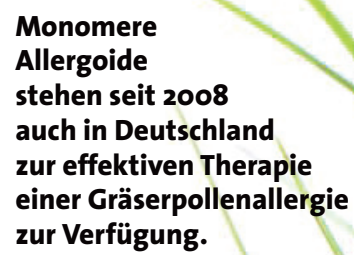

muntherapie mit der Lais ${ }^{\circledR}$ 3-Gräser-Tablette oder einer rein symptomatischen Therapie (Caffarelli C et al. Allergy 2000; 55: 1142-7). Unter der Immuntherapie gingen Symptom- und Medikationsscore während der Pollensaison signifikant im Vergleich zur Kontrollgruppe zurück. Die Therapie war sehr gut verträglich, die Compliance gut, berichtete Eberle.

In einer weiteren Studie mit 40 Kindern mit allergischer Rhinitis bzw. Rhinitis und Asthma (Durchschnittsalter sieben Jahre) hatte sich die Therapie ohne Aufdosierungsphase als wirksam und sicher erwiesen (Agostinis $\mathrm{F}$ et al. Eur Ann Allergy Clin Immunol 2009; 41: 177-80). Während der Behandlungsdauer von zwei Jahren führte die prä- und cosaisonale Therapie zu einer kontinuierlichen Reduktion des Symptomscores.

djb

\section{Symposium ,Mo-}

nomere Allergoide

- ein überlegenes

Therapiekonzept für

SLIT und SCIT?" im

Rahmen von „Aller-

gologie im Kloster“.

Kloster Eberbach, 30.

April 2010. Veranstal-

ter: Lofarma

Deutschland, Willich

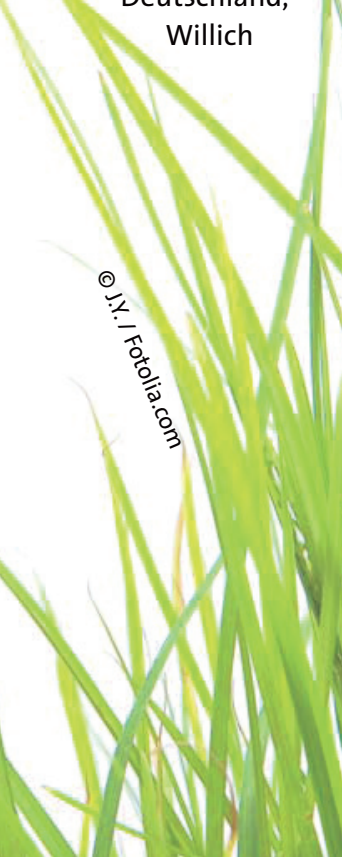

Heuschnupfen oder nicht?

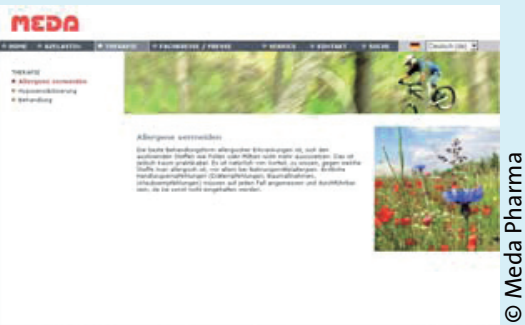

Unter der Adresse www.azelastine.info hat das Unternehmen Meda für Patienten mit Heuschnupfen einen Anlaufpunkt im Internet eingerichtet. Die Website informiert über das Antihistaminikum Azelastin, aber auch allgemein und laienverständlich über die Diagnostik und Therapie der allergischen Rhinitis. Aktuell neu hinzugekommen ist im Bereich „Service“ ein Selbsttest-Fragebogen, mit dessen Hilfe die Wahrscheinlichkeit einer Heuschnupfen-Diagnose abgeklärt werden kann. Auf Grundlage der Ergebnisse kann ein Arzt konsultiert werden, der dann die geeignete Behandlung in die Wege leitet.

Nach Informationen von Meda Pharma, Bad Homburg

\section{Spielerische Ateminfo}

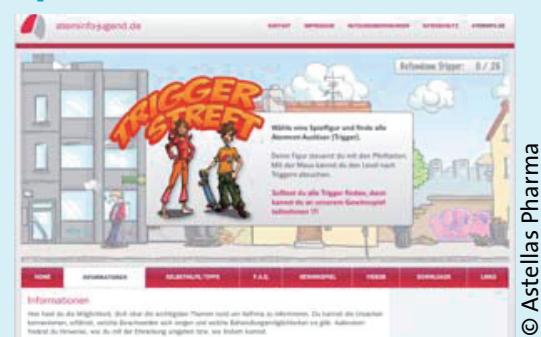

Im Mai ist die speziell auf jugendliche Asthmatiker ausgerichtete Website www. ateminfo-jugend.de ans Netz gegangen. Im Mittelpunkt steht ein E-Learning-Spiel, bei dem man interaktiv sein Wissen über die Erkrankung erweitern kann. Dazu geht man gemeinsam mit einer der Spielfiguren auf die Suche nach Asthmatriggern. Neben Videos zur Bedienung und Reinigung des Novolizer ${ }^{\circledR}$ enthält die Website im Bereich „Informationen“ auch alle wichtigen Fakten zum Krankheitsbild, wie Ursachen, Beschwerden, Behandlung, Prävention und Linderung. Wichtige Tipps zum Verhalten im Notfall oder zu Asthma und Sport finden sich unter ,Selbsthilfe/ Tipps“. Verschiedene Downloads zur Ersthilfe, für ein elektronisches Asthmatagebuch und Anleitungen zu diversen Inhalatoren runden das Service-Angebot $a b$.

Nach Informationen von Astellas Pharma, München 